\title{
A One-pot Multi-component Synthesis of Dihydropyrimidinone/Thione and Dihydropyridine Derivatives via Biginelli and Hantzsch Condensations using $t$-BuOK as a Catalyst Under Solvent-free Conditions
}

\author{
Abdelmadjid Debache ${ }^{*}, \mathrm{a}$, Louisa Chouguiat ${ }^{\mathrm{a}}$, Raouf Boulcina ${ }^{\mathrm{a}}$ and Bertrand Carboni ${ }^{\mathrm{b}}$ \\ ${ }^{a}$ Laboratoire des Produits Naturels d'Origine Végétale et de Synthèse Organique, Département de Chimie, Faculté des \\ Sciences Exactes, Université Mentouri de Constantin, 25000 Constantine, Algérie \\ ${ }^{b}$ Sciences Chimiques de Rennes, UMR 6226 CNRS-Université de Rennes 1, Campus de Beaulieu, 35042 Rennes \\ CEDEX, France
}

\begin{abstract}
The synthesis of various substituted Biginelli 3,4-dihydropyrimidinone/thione and Hantzsch 1,4dihydropyridine derivatives has been achieved using a modified procedure in the presence of potassium ter-butoxide $(t-$ $\mathrm{BuOK}$ ) as a catalyst under solvent-free conditions, in good to excellent yields.
\end{abstract}

Keywords: $t$-BuOK, Biginelli 3,4-dihydropyrimidinones/thiones, Hantzsch 1,4-dihydropyridines, one-pot condensation, solvent-free conditions.

\section{INTRODUCTION}

Dihydropyridines (DHPs) have attracted increasing interest due to their diverse therapeutic and pharmacological properties such as insecticidal, bactericidal and herbicidal effects [1]. DHP drugs, namely nifedipine, nicardipine and amlodipine, are cardiovascular agents for the treatment of hypertension [2]. A number of DHP calcium antagonists have been introduced as potential drugs for the treatment of congestive heart failure [3]. In addition, dihydropyridines find applications in stereo specific hydrogen transfer reduction of phenylglyoxylic and pyruvic acid to biomimetic models of lactase dehydrogenase [4]. Recently, DHPs are used as organocatalysts for asymmetric reactions such as hydrogenation of quinolines in the synthesis of alkaloids [5], asymmetric reductive amination of aldehydes [6] and hydrogenation of $\alpha, \beta$-unsaturated aldehydes and ketones [7]. Classical Hantzsch synthesis of these compounds is carried out in acetic acid or by refluxing in alcohol for a long time [8]. Several other methods are reported including use of microwaves [9], molecular iodine [10], cyanuric chloride [11], ionic liquids [12], silica gel/NaHSO 4 [13], TMSCl-NaI [14], metal triflates [15] and ultrasound irradiations [16].

Additionally, dihydropyrimidinones (DHPMs) have exhibited important therapeutic and pharmacological properties as the integral backbone of several calcium channel blockers [17], antihypertensive agents [18], and $\alpha 1 \mathrm{a}$-antagonists [19a]. A broad range of biological effects including antiviral, antitumor, antibacterial and anti-inflammatory activities has

\footnotetext{
*Address correspondence to this author at the Laboratoire des Produits Naturels d'Origine Végétale et de Synthèse Organique, Département de Chimie, Faculté des Sciences Exactes, Université Mentouri de Constantine, 25000 Constantine, Algérie; Tel/Fax: 213318188 62;

E-mail: a_debache@yahoo.fr
}

been described for these compounds $[20,19 b, c]$. Some of the representative compounds of this class possess antiviral, antibacterial, antihypertensive and antitumor activities [21, 19d]. Several alkaloids isolated from marine sources also exhibit interesting biological activities, molecular structures of which contain the dihydropyrimidinone moiety [20]. Therefore, their synthesis has been the focus of great interest for organic and medicinal chemists [21]. The original Biginelli protocol for the preparation of DHPMs consisted of heating a mixture of three components which included $\beta$ ketoester, aldehyde and urea in ethanol containing a catalytic amount of $\mathrm{HCl}$ [22]. The major drawbacks associated with this protocol are the use of strong acid as well as the low yields in the case of substituted aromatic and aliphatic aldehydes. To enhance the efficiency of the Biginelli reaction, various catalysts and reaction conditions have been studied including classical conditions with ultrasound [23] or microwave-assisted irradiations [24], solid-support [25], ionic liquids [26], Lewis acid catalysts such as $\mathrm{LiBr}$ [27], $\mathrm{NH}_{4} \mathrm{Cl}$ [28], $\mathrm{MgBr}_{2}$ [29], $\mathrm{CaF}_{2}$ [30], $\mathrm{FeCl}_{3} \cdot 6 \mathrm{H}_{2} \mathrm{O}$ [31], $\mathrm{Mn}(\mathrm{OAc})_{3}$ [32], $\mathrm{InBr}_{3}$ [33], $\mathrm{ZnI}_{2}$ [34], $\mathrm{CdCl}_{2}$ [35], $\mathrm{H}_{3} \mathrm{BO}_{3}$ [36], $\mathrm{PhB}(\mathrm{OH})_{2}$ [37] and $\mathrm{CuI}$ [38]. The catalytic effect of metal cations is even more pronounced with methods based on metal salts with non-nucleophilic anions such as $\mathrm{LiClO}_{4}$ [39], $\mathrm{CuSO}_{4} .5 \mathrm{H}_{2} \mathrm{O}$ [40], $\mathrm{Cu}(\mathrm{OTf})_{2}$ [41], $\mathrm{Al}\left(\mathrm{HSO}_{4}\right)_{3}$ [42], trimethylsilyl triflate [43], which allow the preparation of DHPMs in good to high yields. The use of $\mathrm{BF}_{3} \cdot \mathrm{OEt}_{2}$ [44], polyphosphate ester [45], $\left(\mathrm{NH}_{4}\right)_{2} \mathrm{CO}_{3}$ [46], $\mathrm{NaCl}$ [47] and $\left\{\mathrm{Fe}_{2} \mathrm{CuO}\right\}$ clusters [48] has also been described. The Biginelli reaction can strongly be accelerated by various procedures including heteropoly acids [49], silica sulfuric acid [50] and ferric chloride/tetraethyl orthosilicate [51].

On the other hand, a number of the reported protocols to synthesize DHPs and DHPMs requiring solvents and catalysts are not acceptable in the context of green synthesis; 
utilize reagents and catalysts which are either toxic or expensive and stoichiometric use of reagents with respect to reactant. Also, the clean handling of some anhydrous metal halides is not easy enough in the laboratory apart from their hygroscopic nature due to strong tendency for hydrolysis. However, the developments in this area demand further searches for better catalysts that could be superior to the existing ones with regard to toxicity, handling, and recyclability. In this respect, we are interested to introduce potential catalysts to overcome these limitations.

\section{RESULTS AND DISCUSSION}

Recently, we have reported that Biginelli and Hantzsch condensation reactions are facilitated in the presence of a catalytic amount of triethylamine and triphenylphosphine as Brønsted and Lewis bases respectively [52]. Very recently, Z-L. Shen and co-workers [53] reported that $t$-BuOK catalyzes one-pot synthesis of DHPMs via a Biginelli-type condensation from aldehydes and 2-phenylacetophenone.

A literature survey clearly shows that there is no report on the application of $t$-BuOK as Brønsted base catalyst for classic Biginelli and Hantzsch condensation reactions. Here, we wish to report the capacity of potassium tet-Butoxide as potential Brønsted base catalyst for the one-pot synthesis of 3,4-dihydropyrimidinones and 1,4-dihydropyridines and their analogues via solvent-free Hantzsch and Biginelli condensation protocols, respectively.

In the efforts to develop an efficient and environmentally benign method for the synthesis of DHPs and DHPMs we initiated our study with the base-catalyzed Hantzsch condensation by subjecting catalytic amount of $t$-BuOK to the mixture of 2-furaldehyde ( 1 equiv.), which usually gives good yields of the corresponding product, ethyl acetoacetate (2 equiv.) and ammonium acetate (2 equiv.) in ethanol at room temperature. Unfortunately, the resulted yield was very poor even after $24 \mathrm{~h}$ of stirring. To see the effect of reaction, various solvent systems were tested at different temperatures. We found that the synthesis of DHP $\mathbf{4 f}$ was efficiently catalyzed by $t$-BuOK in solvent free condition at elevated temperature leading to a good yield of product (Table 1, entry 6). The reaction condition was then optimized by conducting the reaction in different temperatures and employing different catalyst loadings. The different experiments show that the best result was obtained by the application of $10 \mathrm{~mol} \%$ of $t-$ $\mathrm{BuOK}$ in solvent free condition at $60^{\circ} \mathrm{C}$ (Scheme 1). Other amounts of the catalyst substantially reduced the yield as side products were formed.

In order to study the scope and generality of this methodology, a variety of substituted aromatic aldehydes were subjected to the previous reaction with 2-furaldehyde. Unfortunately, it was observed that even under optimized conditions, that the corresponding 1,4-DHPs were isolated in very moderate yields due to the formation of many other side products, also, the starting material 2a was still present in the crude products (according to ${ }^{1} \mathrm{H}$ NMR spectra). In comparison, good yield was obtained when 2-thiophenecarboxaldehyde was employed (Table 1, entry 5). However, the reaction of other aromatic aldehydes led to lower yields.

After many trials, we decided to employ other more reactive $\beta$-ketoesters such as dimedone 2d (5,5dimethylcyclohexan-1,3-dione) as a second equivalent with ethyl acetoacetate. Thus, the reaction of benzaldehyde (1 equiv.), dimedone (1 equiv.), ethyl acetoacetate (1 equiv.) and ammonium acetate (2 equiv.) in the presence of 10 $\mathrm{mol} \%$ of $t$-BuOK in solvent free condition at $60^{\circ} \mathrm{C}$ affords after only $2 \mathrm{~h}$, the corresponding polyhydroquinoline in excellent yield (Scheme 2). So, the reaction was amenable to a wide range of aromatic and heteroaromatic aldehydes and

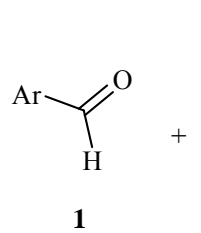

1<smiles>CCOC(=O)CC(C)=O</smiles>

2a

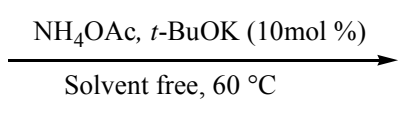<smiles>CCOC(=O)C1=C(C)NC(C)=C(C(=O)OCC)C1[Al]</smiles>

4a-f

Scheme (1).

Table 1. $\quad t$-BuOK-Catalyzed Hantzsch Synthesis of 1,4-dihydropyridines Under Optimized Reaction Conditions ${ }^{\mathrm{a}}$

\begin{tabular}{|c|c|c|c|c|c|c|}
\hline \multirow{2}{*}{ Entry } & DHP & Ar & \multirow{2}{*}{ Time (h) } & Yield (\%) & \multicolumn{2}{|c|}{ M.p. $\left({ }^{\circ} \mathbf{C}\right)$} \\
\cline { 3 - 6 } & & & & Measured & Reported \\
\hline \hline 1 & $4 \mathrm{a}$ & $\mathrm{C}_{6} \mathrm{H}_{5}$ & 5 & 48 & $158-160$ & $158-160[54 \mathrm{a}]$ \\
\hline 2 & $4 \mathrm{~b}$ & $2-\left(\mathrm{OCH}_{3}\right)-\mathrm{C}_{6} \mathrm{H}_{4}$ & 10 & 23 & $141-143$ & $140-142[54 \mathrm{~b}]$ \\
\hline 3 & $4 \mathrm{c}$ & $4-\left(\mathrm{NO}_{2}\right)-\mathrm{C}_{6} \mathrm{H}_{4}$ & 6 & 36 & $162-164$ & $162-164[54 \mathrm{a}]$ \\
\hline 4 & $4 \mathrm{~d}$ & $3-\left(\mathrm{NO}_{2}\right)-\mathrm{C}_{6} \mathrm{H}_{4}$ & 8 & 31 & $1729-131[54 \mathrm{a}]$ \\
\hline 5 & $4 \mathrm{e}$ & $2-$-thienyl & 2 & 80 & $171-173$ & $171-173[54 \mathrm{a}]$ \\
\hline 6 & $4 \mathrm{f}$ & $2-f u r y l$ & 3 & 84 & $160-162$ & $160-161[54 \mathrm{a}]$ \\
\hline
\end{tabular}

a: The reactions were carried out at $60^{\circ} \mathrm{C}$ using aldehyde $(2 \mathrm{mmol})$, ethyl acetoacetate $(2 \mathrm{mmol})$, dimedone $(2 \mathrm{mmol})$ ammonium acetate $(4 \mathrm{mmol})$ and $t$-BuOK $(0.2 \mathrm{mmol})$ under solvent-free conditions. ${ }^{\text {b }}$ : Yields were measured by ${ }^{1} \mathrm{H}$ NMR spectra. 
gave in all cases the desired products in very good yields and the results are summarized in Table 2. It is noteworthy that the reactions proceeded at a faster rate with electron donating aldehydes (entries 1-4) and were slightly slow with electron withdrawing ones (entry 6) with the exception of polyhydroquinoline $4 \mathbf{k}$ (entry 5) which gave the desired product in excellent yield. Good yields were also achieved for heterocyclic aldehydes such as 2-furaldehyde (entry 7). However, aliphatic aldehydes such as acetaldehyde and isobutyraldehyde afforded very poor results.

In addition, we also investigated the reactions using 2methoxybenzaldehyde or 4-methoxybenzaldehyde with two equivalents of dimedone as a $\beta$-dicarbonyl compound under the above optimized reaction conditions. As described in experimental part, the corresponding products (4o and $\mathbf{4 p}$ ) were obtained in good yields [55].

In a typical procedure, $2 \mathrm{mmol}$ of aldehyde, $2 \mathrm{mmol}$ of dimedone, $2 \mathrm{mmol}$ of ethyl acetoacetate and $4 \mathrm{mmol}$ of ammonium acetate were mixed in solvent free condition in the presence of $0.2 \mathrm{mmol}$ of $t-\mathrm{BuOK}$ and the reaction mixture was stirred for $1-7 \mathrm{~h}$ at $60^{\circ} \mathrm{C}$, after work-up, it produced the corresponding polyhydroquinolines in good yields.

A plausible mechanism of the $t$-BuOK catalyzed Hantzsch condensation is shown in Scheme (3) based on the previous reports, our observations and obtained results.

Encouraged by these results, we next focused our efforts to study the effect of $t$-BuOK on the Biginelli condensation reaction (Scheme 4). In order to achieve the optimum conditions, the reaction of benzaldehyde 1a with ethyl acetoacetate 2a and urea 3a was selected as a model. We began our trials by comparing the catalytic efficiency of $t$ - BuOK with traditional Lewis acid. The reactions proceeded in moderate to good yields. The effect of the catalyst amount on the synthesis of DHPM 5a was also investigated and the results show that the reactants hardly react when no catalyst is added, and the yield of Biginelli product is up to $70 \%$ with modifying the amount of $t$-BuOK from 20 to $10 \%$ mol. It was observed that similar results were obtained by decreasing the amount of the catalyst to $50 \% \mathrm{~mol}$. The effect of different solvents was then studied. In water, the reactants do not react well, probably owing to the lower solubility of reactants in water. In ethanol, $\mathrm{CH}_{3} \mathrm{CN}$ or THF, Biginelli product is obtained in moderate to good yields. However, the reaction at $80^{\circ} \mathrm{C}$, under solvent-free condition gave very good yield of the desired product (Table 3, entry 1).

With these results in hand, we set out to examine the scope of this reaction. As shown in Table 3, different reactants are employed under the same conditions as entry 1 under smooth reactions, and the corresponding DHPMs were isolated in good to excellent yields. The results show that the generality of the present protocol is effective for both ethyl acetoacetate 2a and 1,4-pentanedione $\mathbf{2 b}$ of different aldehydes. For aromatic aldehydes carrying either electrondonating or electron-withdrawing substituents, the products are obtained in high yields (entries 1-8). By replacing urea with thiourea $\mathbf{3 b}$ in the reaction system under the proportion of 1:1:1.5 (benzaldehyde: ethyl acetoacetate : thiourea), the corresponding Biginelli products are also obtained in high yields (entries 9 and 10).

Next, we investigated the effect of others $\beta$-ketoesters such as 1,3-cyclohexanedione 2c or 5,5-dimethyl-1,3cyclohexanedione 2d (Scheme 5). Aromatic aldehydes such as benzaldehyde and different substituted benzaldehydes<smiles>CCOC(=O)CC(C)=O</smiles><smiles>CCOC(=O)C1=C(C)NC2=C(C(=O)CC(C)(C)C2)C1[Al]</smiles>

4g-n

Scheme (2).

Table 2. $\quad t$-BuOK-Catalyzed Hantzsch Synthesis of Polyhydroquinolines Under Optimized Reaction Conditions ${ }^{\mathrm{a}}$

\begin{tabular}{|c|c|c|c|c|c|c|}
\hline \multirow{2}{*}{ Entry } & \multirow{2}{*}{ DHP } & \multirow{2}{*}{ Ar } & \multirow{2}{*}{ Time (h) } & \multirow{2}{*}{ Yield $^{\mathrm{b}}(\%)$} & \multicolumn{2}{|c|}{ M.p. $\left({ }^{\circ} \mathrm{C}\right)$} \\
\hline & & & & & Measured & Reported \\
\hline 1 & $4 \mathrm{~g}$ & $\mathrm{C}_{6} \mathrm{H}_{5}$ & 2 & 73 & $205-207$ & $202-204[56 a]$ \\
\hline 2 & $4 \mathrm{~h}$ & 4- $\left(\mathrm{CH}_{3}\right)-\mathrm{C}_{6} \mathrm{H}_{4}$ & 2 & 92 & $260-262$ & $260-262[56 a]$ \\
\hline 3 & $4 \mathrm{i}$ & $4-\left(\mathrm{CH}_{3} \mathrm{O}\right)-\mathrm{C}_{6} \mathrm{H}_{4}$ & 1 & 85 & $256-258$ & $255-257[56 a]$ \\
\hline 4 & $4 j$ & $4-(\mathrm{OH})-\mathrm{C}_{6} \mathrm{H}_{4}$ & 1 & 94 & $232-234$ & $232-234[56 a]$ \\
\hline 5 & $4 \mathrm{k}$ & 4-(Br)- $\mathrm{C}_{6} \mathrm{H}_{4}$ & 2 & 94 & $251-253$ & $253-254[56 a]$ \\
\hline 6 & 41 & $4-\left(\mathrm{NO}_{2}\right)-\mathrm{C}_{6} \mathrm{H}_{4}$ & 7 & 90 & $240-242$ & $242-244[56 b]$ \\
\hline 7 & $4 \mathrm{~m}$ & 2-furyl & 3 & 72 & $246-248$ & $248-249[56 c]$ \\
\hline 8 & $4 n$ & Styryl & 3 & 65 & $206-208$ & $206-207[56 c]$ \\
\hline
\end{tabular}

a: The reactions were carried out at $60^{\circ} \mathrm{C}$ using aldehyde $(2 \mathrm{mmol})$, ethyl acetoacetate $(2 \mathrm{mmol})$, dimedone $(2 \mathrm{mmol})$, ammonium acetate $(4 \mathrm{mmol})$ and $t$-BuOK $(0.2 \mathrm{mmol})$ under solvent-free conditions. ${ }^{b}$ : Isolated yields. 


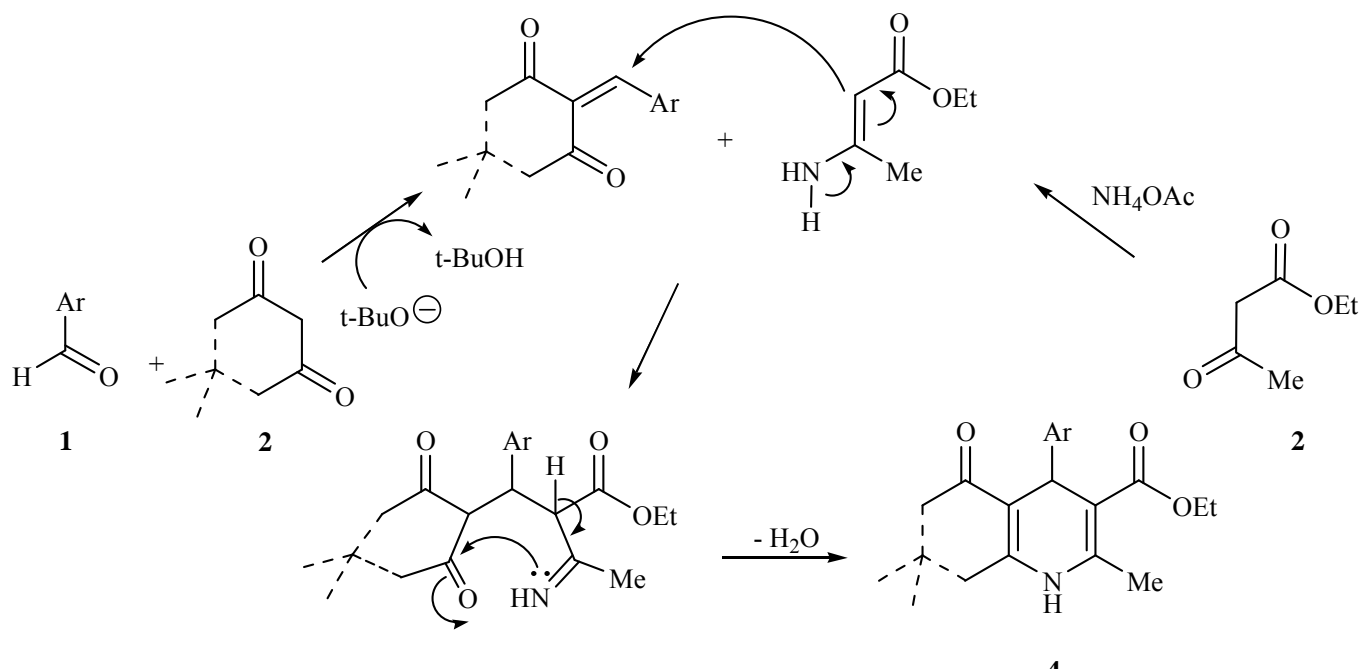

Scheme (3).<smiles>[R]C(=O)CC(C)=O</smiles>

Scheme (4).

Table 3. t-BuOK-Catalyzed Biginelli Synthesis of 3,4-dihydropyrimidinones Under Optimized Reaction Conditions ${ }^{\mathrm{a}}$

\begin{tabular}{|c|c|c|c|c|c|c|c|c|}
\hline Entry & DHPM & Ar & $\mathbf{R}$ & $\mathbf{X}$ & Time (h) & Yield $^{\mathrm{b}}(\%)$ & \multicolumn{2}{|c|}{ M.p. $\left({ }^{\circ} \mathrm{C}\right)$} \\
\hline 2 & $5 b$ & 4- $\left(\mathrm{CH}_{3}\right)-\mathrm{C}_{6} \mathrm{H}_{4}$ & OEt & $\mathrm{O}$ & 3 & 81 & $212-214$ & $215-216[57 a]$ \\
\hline 3 & $5 c$ & $3-\left(\mathrm{CH}_{3}\right)-\mathrm{C}_{6} \mathrm{H}_{4}$ & OEt & $\mathrm{O}$ & 8 & 65 & $210-212$ & $208-209$ [52c] \\
\hline 5 & $5 e$ & $2-(\mathrm{OH})-\mathrm{C}_{6} \mathrm{H}_{4}$ & OEt & $\mathrm{O}$ & 6 & 59 & $203-205$ & $202-203[36]$ \\
\hline 6 & $5 \mathrm{f}$ & Styryl & OEt & $\mathrm{O}$ & 5 & 54 & $223-225$ & $223-225$ [52c] \\
\hline 7 & $5 \mathrm{~g}$ & $\mathrm{C}_{6} \mathrm{H}_{5}$ & $\mathrm{Me}$ & $\mathrm{O}$ & 8 & 68 & $236-238$ & $233-235[57 b]$ \\
\hline 8 & $5 \mathrm{~h}$ & $4-\left(\mathrm{CH}_{3}\right)-\mathrm{C}_{6} \mathrm{H}_{4}$ & $\mathrm{Me}$ & $\mathrm{O}$ & 8 & 76 & $202-204$ & $203-205[57 b]$ \\
\hline
\end{tabular}

${ }^{a}$ : The reactions were carried out at $80^{\circ} \mathrm{C}$ using aldehyde $(2 \mathrm{mmol}), \beta$-ketoester $(2 \mathrm{mmol})$, urea or thiourea $(3 \mathrm{mmol})$ and $t$-BuOK $(0.2 \mathrm{mmol})$ under solvent-free conditions. ${ }^{b}:$ Isolated yields.

react with dimedone and urea in the presence of $t$-BuOK to afford the corresponding octahydroquinazolines in excellent yields in reduced reaction time compared with ethyl acetoacetate (Table 4).

The mechanism of this multicomponent reaction is similar to the one previously reported in papers for the Biginelli reaction; the formation of product $\mathbf{5}$ may involve an acylimine intermediate, the addition of $\beta$-ketoester to the iminium ion, and subsequent cyclization and dehydration (Scheme 6).

\section{CONCLUSION}

In conclusion, we have successfully developed an easy and efficient method to prepare a variety of 4-substituted1,4-dihydropyridine and 3,4-dihydropyrimidinone derivatives from the reaction of different aromatic or heteroaromatic aldehydes, $\beta$-ketoesters and ammonium acetate or urea in the presence of catalytic amount of $t$-BuOK under solvent- 
<smiles></smiles>

1<smiles>[R]C1([R])CC(=O)CC(=O)C1</smiles>

2c-d<smiles>NC(N)=O</smiles>

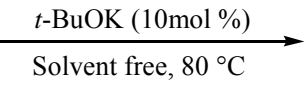

3a<smiles>[R]C1([R])CC(=O)C2=C(C1)NC(=O)NC2[Al]</smiles>

$5 k-r$

Scheme (5).

Table 4. $\quad t$-BuOK-Catalyzed Biginelli Synthesis of Octahydroquinazolines Under Optimized Reaction Conditions ${ }^{\mathrm{a}}$

\begin{tabular}{|c|c|c|c|c|c|c|c|c|}
\hline \multirow{2}{*}{ Entry } & \multirow{2}{*}{ DHPM } & \multirow{2}{*}{ Ar } & \multirow{2}{*}{$\mathbf{R}^{1}$} & \multirow{2}{*}{$\mathbf{R}^{2}$} & \multirow{2}{*}{ Time (h) } & \multirow{2}{*}{ Yield $^{\mathrm{b}}(\%)$} & \multicolumn{2}{|c|}{ M.p. $\left({ }^{\circ} \mathrm{C}\right)$} \\
\hline & & & & & & & Measured & Reported \\
\hline 1 & $5 \mathrm{k}$ & $\mathrm{C}_{6} \mathrm{H}_{5}$ & $\mathrm{Me}$ & $\mathrm{Me}$ & 5 & 76 & $288-290$ & $287-290[58 \mathrm{a}]$ \\
\hline 2 & 51 & $4-\left(\mathrm{CH}_{3}\right)-\mathrm{C}_{6} \mathrm{H}_{4}$ & $\mathrm{Me}$ & $\mathrm{Me}$ & 6 & 66 & $>300$ & $>300[58 \mathrm{a}]$ \\
\hline 3 & $5 \mathrm{~m}$ & 4- $\left(\mathrm{CH}_{33} \mathrm{O}\right)-\mathrm{C}_{6} \mathrm{H}_{4}$ & $\mathrm{Me}$ & $\mathrm{Me}$ & 4 & 67 & $241-243$ & - \\
\hline 7 & $5 n$ & 4-(Br)- $\mathrm{C}_{6} \mathrm{H}_{4}$ & $\mathrm{Me}$ & $\mathrm{Me}$ & 8 & 79 & $>300$ & $>300[58 \mathrm{a}]$ \\
\hline 4 & 50 & $\mathrm{C}_{6} \mathrm{H}_{5}$ & $\mathrm{H}$ & $\mathrm{H}$ & 8 & 68 & $>300$ & $308[58 b]$ \\
\hline 5 & $5 p$ & $4-\left(\mathrm{CH}_{3}\right)-\mathrm{C}_{6} \mathrm{H}_{4}$ & $\mathrm{H}$ & $\mathrm{H}$ & 3 & 62 & $296-298$ & 298 [58b] \\
\hline 6 & $5 q$ & 4- $\left(\mathrm{CH}_{3} \mathrm{O}\right)-\mathrm{C}_{6} \mathrm{H}_{4}$ & $\mathrm{H}$ & $\mathrm{H}$ & 5 & 80 & $282-284$ & $284[58 b]$ \\
\hline 8 & $5 \mathrm{r}$ & 4-(Br)- $\mathrm{C}_{6} \mathrm{H}_{4}$ & $\mathrm{H}$ & $\mathrm{H}$ & 8 & 69 & $295-297$ & 298 [58b] \\
\hline
\end{tabular}

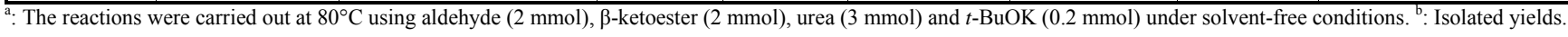

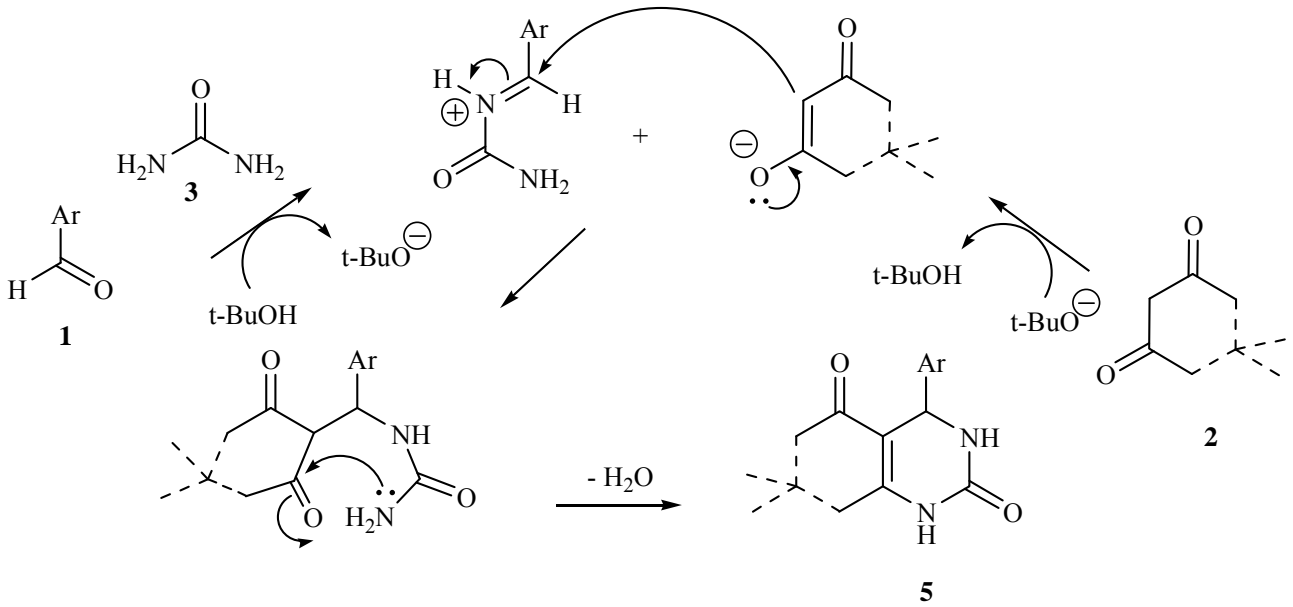

Scheme (6).

free conditions. The catalytic activity of $t$ - $\mathrm{BuOK}$ is notable and the use of low cost, commercially available materials for the synthesis of Hnatzsch and Biginelli products in good to excellent yields is also significant under the aspect of environmentally benign processes. These advantages make $t$ $\mathrm{BuOK}$ as a powerful catalyst for the synthesis of 1,4-DHPs and 3,-DHPMs and their analogs.

\section{EXPERIMENTAL}

4.1. General Procedure for the Synthesis of 1,4dihydropyrines and Polyhydroquinolines 4

Aldehyde ( $2 \mathrm{mmol}), \beta$-ketoester ( $4 \mathrm{mmol}), \mathrm{NH}_{4} \mathrm{OAc}(4$ $\mathrm{mmol})$ and $t$-BuOK $(0.2 \mathrm{mmol})$ were stirred at $60{ }^{\circ} \mathrm{C}$. After completion of the reaction as indicated by TLC, the crude product was purified by recrystallization from ethanol to yield the highly pure Hantzsch 1,4-dihydropyridine derivatives. The physical data (M.p., IR, NMR) of known compounds were found to be identical with those reported in the literature. Spectroscopic data for selected examples are shown below.

\section{Ethyl 4-phenyl-2,6-dimethyl-1,4-dihydropyridine-3,5- dicarboxylate (4a)}

M.p. $158-160^{\circ} \mathrm{C}$, IR (KBr): 3335, 1692, 1651, 1490 , 1239, 1122, $720 \mathrm{~cm}^{-1}$. ${ }^{1} \mathrm{H}$ NMR $\left(250 \mathrm{MHz}, \mathrm{CDCl}_{3}\right) \delta: 7.32-$ $7.14\left(\mathrm{~m}, 5 \mathrm{H}, \mathrm{CH}_{\mathrm{Ar}}\right), 5.71(\mathrm{~s}, 1 \mathrm{H}, \mathrm{NH}), 5.01(\mathrm{~s}, 1 \mathrm{H}, \mathrm{CH}), 4.11$ $\left(\mathrm{q}, J=7.1,4 \mathrm{H}, 2 \mathrm{CH}_{2}\right), 2.36\left(\mathrm{~s}, 6 \mathrm{H}, 2 \mathrm{CH}_{3}\right), 1.24(\mathrm{t}, J=7.1,6 \mathrm{H}$, 
$\left.2 \mathrm{CH}_{3}\right) .{ }^{13} \mathrm{C} \mathrm{NMR}\left(62.5 \mathrm{MHz}, \mathrm{CDCl}_{3}\right) \delta: 167.6,145.4,143.8$, 135.6, 128.4, 127.9, 104.2, 59.7, 39.6, 19.5, 14.2 .

Ethyl 4-(2-thienyl)-2,6-dimethyl-1,4-dihydropyridine-3,5dicarboxylate (4e)

M.p. $171-173^{\circ} \mathrm{C}$, IR (KBr): 3421, 1647, 1512, 1485, $1213,1115,752 \mathrm{~cm}^{-1} ;{ }^{1} \mathrm{H}$ NMR $\left(250 \mathrm{MHz}, \mathrm{CDCl}_{3}\right) \delta: 7.06-$ $6.79\left(\mathrm{~m}, 3 \mathrm{H}, \mathrm{CH}_{\mathrm{Ar}}\right), 6.55(\mathrm{~s}, 1 \mathrm{H}, \mathrm{NH}), 5.35(\mathrm{~s}, 1 \mathrm{H}, \mathrm{CH}), 4.16$ $\left(\mathrm{q}, J=7.0 \mathrm{~Hz}, 4 \mathrm{H}, 2 \mathrm{CH}_{2}\right), 2.32\left(\mathrm{~s}, 6 \mathrm{H}, 2 \mathrm{CH}_{3}\right), 1.28(\mathrm{t}, J=7.0$ $\left.\mathrm{Hz}, 6 \mathrm{H}, 2 \mathrm{CH}_{3}\right) .{ }^{13} \mathrm{C}$ NMR $\left(62.5 \mathrm{MHz}, \mathrm{CDCl}_{3}\right) \delta: 167.5$, $152.1,145.0,143.7,126.3,123.1,103.3,59.9,34.3,19.2$, 14.3 .

Ethyl 4-(2-furyl)-2,6-dimethyl-1,4-dihydropyridine-3,5dicarboxylate (4f)

M.p. $160-162^{\circ} \mathrm{C}$, IR (KBr): 3344, 1701, 1649, 1485, 1371, 1207, 1120, $727 \mathrm{~cm}^{-1} .{ }^{1} \mathrm{H}$ NMR $\left(250 \mathrm{MHz}, \mathrm{CDCl}_{3}\right) \delta$ : 7.22 (s, 1H, NH); 6.22-5.94 (m, 3H, $\left.\mathrm{CH}_{\mathrm{Ar}}\right), 5.20(\mathrm{~s}, 1 \mathrm{H}, \mathrm{CH})$, $4.11\left(\mathrm{q}, J=7.1 \mathrm{~Hz}, 4 \mathrm{H}, 2 \mathrm{CH}_{2}\right), 2.32\left(\mathrm{~s}, 6 \mathrm{H}, 2 \mathrm{CH}_{3}\right), 1.27(\mathrm{t}$, $\left.6 \mathrm{H}, J=7.1 \mathrm{~Hz}, 2 \mathrm{CH}_{3}\right) .{ }^{13} \mathrm{C} \mathrm{NMR}\left(62.5 \mathrm{MHz}, \mathrm{CDCl}_{3}\right) \delta$ : $167.5,158.7,145.2,140.8 ; 110.0,104.4,100.5,59.8,33.3$, $19.4,14.3$.

Ethyl 2,7,7-Trimethyl-5-oxo-4-phenyl-1,4,4a,5,6,7,8,8aoctahydroquinoline-3-carboxylate $(4 \mathrm{~g})$

M.p. 205-207 ${ }^{\circ} \mathrm{C}$, IR (KBr): 3293, 3058, 2958, 1676, $1610 \mathrm{~cm}^{-1} .{ }^{1} \mathrm{H} \mathrm{NMR}\left(\mathrm{CDCl}_{3}, 250 \mathrm{MHz}\right) \delta: 7.75(\mathrm{~s}, 1 \mathrm{H}, \mathrm{NH})$, 7.34-7.08 (m, 5H, $\left.\mathrm{CH}_{\mathrm{Ar}}\right), 5.07(\mathrm{~s}, 1 \mathrm{H}, \mathrm{CH}), 4.10(\mathrm{q}, J=7.1$ $\left.\mathrm{Hz}, 2 \mathrm{H}, \mathrm{CH}_{2}\right), 2.30\left(\mathrm{~s}, 3 \mathrm{H}, \mathrm{CH}_{3}\right), 2.22-2.10\left(\mathrm{~m}, 4 \mathrm{H}, 2 \mathrm{CH}_{2}\right)$, $1.23\left(\mathrm{t}, J=7.1 \mathrm{~Hz}, 3 \mathrm{H}, \mathrm{CH}_{3}\right), 1.04\left(\mathrm{~s}, 3 \mathrm{H}, \mathrm{CH}_{3}\right), 0.93$ (s, $3 \mathrm{H}$, $\left.\mathrm{CH}_{3}\right) \cdot{ }^{13} \mathrm{C}\left(\mathrm{CDCl}_{3}, 63 \mathrm{MHz}\right) \delta: 196.1,167.7,153.5,150.2$, $147.3,144.4,128.0,126.7,126.0,115.5,111.4,105.7,59.8$, $50.8,40.4,36.6,32.6,29.5,27.0,19.0,14.2$.

Ethyl 2,7,7-trimethyl-5-oxo-4-p-tolyl-1,4,4a,5,6,7,8,8aoctahydroquinoline-3-carboxylate (4h)

M.p. $260-262^{\circ} \mathrm{C}$, IR (KBr): 3294, 3058, 2955, 1645, 1610, 1485, $1218 \mathrm{~cm}^{-1} .{ }^{1} \mathrm{H}$ NMR $\left(\mathrm{CDCl}_{3}, 250 \mathrm{MHz}\right) \delta: 7.21$ (d, J=8.1 Hz, 2H, $\left.\mathrm{CH}_{\mathrm{Ar}}\right), 7.01\left(\mathrm{~d}, J=8.1 \mathrm{~Hz}, 2 \mathrm{H}, \mathrm{CH}_{\mathrm{Ar}}\right), 6.87$ (s, 1H, NH), $5.02(\mathrm{~s}, 1 \mathrm{H}, \mathrm{CH}), 4.04$ (q, J=7.1 Hz, 2H, $\mathrm{CH}_{2}$ ), $2.33\left(\mathrm{~s}, 3 \mathrm{H}, \mathrm{CH}_{3}\right), 2.26\left(\mathrm{~s}, 3 \mathrm{H}, \mathrm{CH}_{3}\right), 2.32-2.18(\mathrm{~m}, 4 \mathrm{H}$, $2 \mathrm{CH}_{2}$ ), 1.24 (t, 3H, J=7.1 Hz, $\left.\mathrm{CH}_{3}\right), 1.09$ (s, 3H, $\left.\mathrm{CH}_{3}\right), 0.95$ $\left(\mathrm{s}, 3 \mathrm{H}, \mathrm{CH}_{3}\right) .{ }^{13} \mathrm{C}\left(\mathrm{CDCl}_{3}, 63 \mathrm{MHz}\right) \delta: 195.8,167.6,149.0$, 144.3, 143.7, 135.4, 128.6, 127.8, 127.3, 111.9, 106.1, 77.5, 76.5, 59.8, 50.7, 40.8, 36.1, 32.7, 29.4, 27.1, 19.2, 14.2.

Ethyl 4-(4-methoxyphenyl)-2,7,7-trimethyl-5-oxo1,4,4a,5,6,7,8,8-aoctahydroquinoline-3-carboxylate (4i)

M.p. $256-258^{\circ} \mathrm{C}$, IR (KBr): 3449, 3348, 3101, 2978, 1647, 1489, $1207 \mathrm{~cm}^{-1} .{ }^{1} \mathrm{H}$ NMR $\left(\mathrm{CDCl}_{3}, 250 \mathrm{MHz}\right) \delta: 7.23$ (d, J=6.7 Hz, 2H, $\left.\mathrm{CH}_{\mathrm{Ar}}\right), 6.8(\mathrm{~s}, 1 \mathrm{H}, \mathrm{NH}) ; 6.75$ (d, 2H, J= 6.7, $\left.\mathrm{CH}_{\mathrm{Ar}}\right) ; 5.00(\mathrm{~s}, 1 \mathrm{H}, \mathrm{CH}), 4.08\left(\mathrm{q}, 2 \mathrm{H}, \mathrm{J}=7.1 \mathrm{~Hz}, \mathrm{CH}_{2}\right)$, $3.74\left(\mathrm{~s}, 3 \mathrm{H}, \mathrm{OCH}_{3}\right), 2.35\left(\mathrm{~s}, 3 \mathrm{H}, \mathrm{CH}_{3}\right), 2.28-2.17(\mathrm{~m}, 4 \mathrm{H}$, $\left.2 \mathrm{CH}_{2}\right), 1.23\left(\mathrm{t}, J=7.1 \mathrm{~Hz}, 3 \mathrm{H}, \mathrm{CH}_{3}\right), 1.06\left(\mathrm{~s}, 3 \mathrm{H}, \mathrm{CH}_{3}\right), 0.94$ $\left(\mathrm{s}, 3 \mathrm{H}, \mathrm{CH}_{3}\right) \cdot{ }^{13} \mathrm{C}\left(\mathrm{CDCl}_{3}, 63 \mathrm{MHz},\right) \delta: 195.9,167.6,157.7$, $148.8,143.5,139.7,133.0,128.9,113.2 ; 106.1,59.8 ; 50.7$, 40.8, 35.7, 32.6, 29.5, 27.1, 19.2, 14.2 .

3,3,6,6-tetramethyl-9-(2-metoxyphenyl)-3,4,6,7,9,10hexahydro-2H,5H-acridine-1,8-dione (4o)

M.p. $183-185^{\circ} \mathrm{C}, \mathrm{IR}(\mathrm{KBr}): 3396,1643,1095,1026 \mathrm{~cm}^{-1}$. ${ }^{1} \mathrm{H}$ NMR $\left(250 \mathrm{MHz}, \mathrm{CDCl}_{3}\right) \delta: 8.48(\mathrm{~s}, 1 \mathrm{H}, \mathrm{NH}) ; 7.10-6.78$ $\left(\mathrm{m}, 4 \mathrm{H}, \mathrm{CH}_{\mathrm{Ar}}\right), 5.24(\mathrm{~s}, 1 \mathrm{H}), 3.72\left(\mathrm{~s}, 3 \mathrm{H}, \mathrm{OCH}_{3}\right), 2.35-2.10$ $\left(\mathrm{m}, 8 \mathrm{H}, 4 \mathrm{CH}_{2}\right), 1.25-0.95\left(\mathrm{~m}, 12 \mathrm{H}, 4 \mathrm{CH}_{3}\right) .{ }^{13} \mathrm{C}\left(\mathrm{CDCl}_{3}, 63\right.$ $\mathrm{MHz}$ ) $\delta: 196.0,157.5,129.8,128.9,115.8,113.2,55.2$, 47.0, 46.4, 32.7, 32.5, 31.4, 29.7.

3,3,6,6-tetramethyl-9-(4-metoxyphenyl)-3,4,6,7,9,10hexahydro-2H,5H-acridine-1,8-dione (4p)

M.p. $249-251^{\circ} \mathrm{C}$, IR (KBr): $3423,1643,1045,1014 \mathrm{~cm}^{-1}$. ${ }^{1} \mathrm{H} \mathrm{NMR}\left(\mathrm{CDCl}_{3}, 250 \mathrm{MHz}\right) \delta: 12.00(\mathrm{~s}, 1 \mathrm{H}, \mathrm{NH}) ; 7.02(\mathrm{~d}$, $\left.2 \mathrm{H}, J=8.6 \mathrm{~Hz}, \mathrm{CH}_{\mathrm{Ar}}\right) ; 6.83\left(\mathrm{~d}, J=8.6 \mathrm{~Hz}, 2 \mathrm{H}, \mathrm{CH}_{\mathrm{Ar}}\right) ; 5.51(\mathrm{~s}$, $1 \mathrm{H}) ; 3.78\left(\mathrm{~s}, 3 \mathrm{H}, \mathrm{OCH}_{3}\right) ; 2.44-2.18\left(\mathrm{~m}, 8 \mathrm{H}, 4 \mathrm{CH}_{2}\right) ; 1.24(\mathrm{~s}$, $\left.6 \mathrm{H}, 2 \mathrm{CH}_{3}\right) ; 1.12\left(\mathrm{~s}, 6 \mathrm{H}, 2 \mathrm{CH}_{3}\right) .{ }^{13} \mathrm{C}\left(\mathrm{CDCl}_{3}, 63 \mathrm{MHz},\right) \delta$ : $194.5 ; 172.6 ; 151.2,146.3,129.3,127.6,111.8,55.3,47.0$, 46.4, 32.9, 32.7, 31.2, 29.4 .

4.2. General Procedure for the Synthesis of 3,4dihydropyrimidinones and Octahydroquinazolines 5

A mixture of aldehydes ( $2 \mathrm{mmol}), \beta$-ketoester $(2 \mathrm{mmol})$, urea or thiourea $(3 \mathrm{mmol})$ and $t$-BuOK $(0.2 \mathrm{mmol})$ was heated at $80^{\circ} \mathrm{C}$ for an appropriate time (monitored by TLC). After completion of the reaction, the mixture was cooled to room temperature and the solid was filtered and recrystallized from EtOH to afford the pure products. The products were characterized by their M.p, IR, ${ }^{1} \mathrm{H}$ and ${ }^{13} \mathrm{C}$ NMR spectral data and their melting points were compared with reported values. Data for selected compounds as shown below:

5-(Ethoxycarbonyl)-6-methyl-4-(4-methylphenyl)-3, 4dihydropyrimidin-2(1H)-one (5b)

M.p. $212-214^{\circ} \mathrm{C}$, IR (KBr): $3244,1705,1649,1223 \mathrm{~cm}^{-1}$. ${ }^{1} \mathrm{H}$ NMR (DMSO-d $\left.{ }_{6}, 250 \mathrm{MHz}\right) \delta: 9.17(\mathrm{~s}, 1 \mathrm{H}, \mathrm{NH}), 7.71(\mathrm{~s}$, $1 \mathrm{H}, \mathrm{NH}), 7.12\left(\mathrm{~m}, 4 \mathrm{H}, \mathrm{CH}_{\mathrm{Ar}}\right), 5.11(\mathrm{~s}, 1 \mathrm{H}, \mathrm{CH}), 3.95(\mathrm{q}$, $\left.J=7.1,2 \mathrm{H}, \mathrm{CH}_{2}\right), 2.25\left(\mathrm{~s}, 3 \mathrm{H}, \mathrm{CH}_{3}\right), 1.10\left(\mathrm{t}, J=7.1,3 \mathrm{H}, \mathrm{CH}_{3}\right)$. ${ }^{13} \mathrm{C}$ (DMSO-d $\left.d_{6}, 63 \mathrm{MHz}\right) \delta: 165.8,152.6,148.7,142.3$, $136.8,129.3,126.6,99.9,59.6,54.0,21.1,18.8,14.5$

5-(Methoxycarbonyl)-6-methyl-4-(4-methylphenyl)-3,4dihydropyrimidin-2(1H)-one (5h)

M.p. $203-205^{\circ} \mathrm{C}$, IR (KBr): 3290, 1701, 1620, $1238 \mathrm{~cm}^{-1}$. ${ }^{1} \mathrm{H}$ NMR (DMSO-d, $\left.250 \mathrm{MHz}\right) \delta: 7.80(\mathrm{~s}, 1 \mathrm{H}, \mathrm{NH}), 9.76(\mathrm{~s}$, $1 \mathrm{H}, \mathrm{NH}), 7.28-7.14\left(\mathrm{~m}, 4 \mathrm{H}, \mathrm{CH}_{\mathrm{Ar}}\right), 5.43(\mathrm{~s}, 1 \mathrm{H}, \mathrm{CH}), 2.36(\mathrm{~s}$, $\left.3 \mathrm{H}, \mathrm{CH}_{3}\right), 2.35\left(\mathrm{~s}, 3 \mathrm{H}, \mathrm{CH}_{3}\right), 2.13\left(\mathrm{~s}, 3 \mathrm{H}, \mathrm{CH}_{3}\right) .{ }^{13} \mathrm{C}$ (DMSO$d_{6}, 63 \mathrm{MHz}, \delta$ : 195.3, 153.1, 145.8, 139.8, 138.1, 129.7, $126.5,110.5,55.7,30.3,21.1,19.7$.

5-(Ethoxycarbonyl)-6-methyl-4-phenyl-3, dihydropyrimidin-2(1H)-thione (5i)

M.p. $224-226^{\circ} \mathrm{C}, \mathrm{IR}(\mathrm{KBr}): 3229,1670,1176,725 \mathrm{~cm}^{-1} .{ }^{1} \mathrm{H}$ NMR (DMSO-d, $250 \mathrm{MHz}) \delta: 10.34$ (s, 1H, NH), 9.76 (s, $1 \mathrm{H}, \mathrm{NH}), 7.50-7.21\left(\mathrm{~m}, 5 \mathrm{H}, \mathrm{CH}_{\mathrm{Ar}}\right), 5.19(\mathrm{~d}, 1 \mathrm{H}, J=7.0, \mathrm{CH})$, $4.01\left(\mathrm{q}, J=7.0,2 \mathrm{H}, \mathrm{CH}_{2}\right), 2.36\left(\mathrm{~s}, 3 \mathrm{H}, \mathrm{CH}_{3}\right), 1.09$ (t, $J=7.0$, $\left.3 \mathrm{H}, \mathrm{CH}_{3}\right) .{ }^{13} \mathrm{C}$ (DMSO-d, $\left.63 \mathrm{MHz}\right) \delta: 174.7,165.6,145.4$, $143.9 ; 129.0 ; 128.1,127.8,126.8,101.2,60.0$, 54.5, 17.6, 14.4 .

\section{7,7-dimethyl-4-phenyl-3,4,7,8-tetrahydroquinazoline- 2,5(1H,6H)-dione (5k)}

M.p: $288-290^{\circ} \mathrm{C}$, IR (KBr): $3240,1701,1634,1221 \mathrm{~cm}^{-1}$. ${ }^{1} \mathrm{H}$ NMR (DMSO-d $d_{6}, 250 \mathrm{MHz}$, $\delta:$ 7.97-7.24 (m, 5H, $\mathrm{CH}_{\mathrm{Ar}}$ ), 6.79 (s, 1H, NH), $5.96(\mathrm{~s}, 1 \mathrm{H}, \mathrm{NH}), 4.31(\mathrm{~s}, 1 \mathrm{H}, \mathrm{CH}), 2.22-$ $2.34\left(\mathrm{~m}, 4 \mathrm{H}, 2 \mathrm{CH}_{2}\right), 1.02-1.13\left(\mathrm{~s}, 6 \mathrm{H}, 2 \mathrm{CH}_{3}\right) .{ }^{13} \mathrm{C}$ (DMSO- 
$\left.d_{6}, 63 \mathrm{MHz}\right) \delta: 195.6,167.1,145.2,141.3,127.7,126.8$, 125.4, 114.8, 101.1, 46.9, 33.1, 32.8, 31.6, 28.2, 26.9.

\section{7,7-dimethyl-4-(p-tolyl)-3,4,7,8-tetrahydroquinazoline- 2,5(1H,6H)-dione (5l)}

M.p. $>300^{\circ} \mathrm{C}$, IR $(\mathrm{KBr}): 3244,1705,1649,1223 \mathrm{~cm}^{-1}$. ${ }^{1} \mathrm{H}$ NMR (DMSO-d 6 , $\left.250 \mathrm{MHz}\right) \delta: 9.49$ (s, 1H, NH), 7.76 (s, $1 \mathrm{H}, \mathrm{NH}), 7.20-6.89\left(\mathrm{~m}, 4 \mathrm{H}, \mathrm{CH}_{\mathrm{Ar}}\right), 5.13(\mathrm{~s}, 1 \mathrm{H}), 2.54-1.89$ $\left(\mathrm{m}, 7 \mathrm{H}, \mathrm{CH}_{3}, 2 \mathrm{CH}_{2}\right), 1.02\left(\mathrm{~s}, 3 \mathrm{H}, \mathrm{CH}_{3}\right) \cdot{ }^{13} \mathrm{C}$ (DMSO-d 6.63 MHz) $\delta: 193.3,163.2,152.5 ; 149.5,142.2,136.6,128.7$, $126.8,108.0,52.1,50.4,32.6,29.2,27.2,21.1$.

\section{CONFLICT OF INTEREST}

None Declared.

\section{ACKNOWLEDGEMENTS}

None Declared.

\section{REFERENCES}

[1] Khadilkar, B.; Borkar, S. Silica gel supported ferric nitrate: A convenient oxidizing reagent. Synth. Commun., 1998, 28, 207-212.

[2] (a) Buhler, F.R.; Kiowski, W. Calcium antagonists in hypertension. J. Hypertens. Suppl., 1987, S3 10; (b) Zolfigol, M.A.; Salehi, P.; Safaiee M. An efficient and eco-friendly procedure for the synthesis of Hantzsch ethyl 1,4-dihydro-2,6-dimethylpyridine-3,5dicarboxylates under mild and green conditions. Lett. Org. Chem., 2006, 3, 153-156.

[3] Vo, D.; Matowe, W.C.; Ramesh, M.; Iqbal, N.; Wolowyk, M.W.; Howlett, S.E.; Knaus, E.E. Syntheses, calcium channel agonistantagonist modulation activities, and voltage-clamp studies of isopropyl 1,4-dihydro-2,6-dimethyl-3-nitro-4-pyridinylpyridine-5carboxylate racemates and enantiomers. J. Med. Chem., 1995, 38, 2851-2859.

[4] Krechl, J.; Smrčková, S. Biomimetic models of lactate dehydrogenase. Tetrahedron Lett., 1989, 30, 5315-5318.

[5] Rueping, M.; Antonchick, A.P.; Theissmann, T. A Highly enantioselective brønsted acid catalyzed cascade reaction: organocatalytic transfer hydrogenation of quinolines and their application in the synthesis of alkaloids. Angew. Chem. Int. Ed. Engl., 2006, 45, 3683-3686.

[6] Hoffmann, S.; Nicoletti, M.; List, B. Catalytic asymmetric reductive amination of aldehydes via dynamic kinetic resolution. J. Am. Chem. Soc., 2006, 128, 13074-13075.

[7] (a) Yang, J.W.; Fonseca, M.T.H.; List, B. A metal-free transfer hydrogenation: Organocatalytic conjugate reduction of $\alpha, \beta$ unsaturated aldehydes. Angew. Chem. Int. Ed. Engl., 2004, 43, 6660-6662; (b) Martin, N.J.A.; List, B. Highly enantioselective transfer hydrogenation of $\alpha, \beta$-unsaturated ketones. J. Am. Chem. Soc., 2006, 128, 13368-13369.

[8] Love, B.; Snader, K.M. The Hantzsch Reaction. I. oxidative dealkylation of certain dihydropyridines. J. Org. Chem., 1965, 30, 1914-1916.

[9] (a) Anniappan, M.; Muralidharan, D.; Perumal, P.T. Synthesis of Hantzsch 1,4-dihydropyridines under microwave irradiation. Synth. Commun., 2002, 32, 659-663; (b) Khadikar, B. M.; Gaikar, V. G.; Chitnavis, A. A. Aqueous hydrotrope solution as a safer medium for microwave enhanced hantzsch dihydropyridine ester synthesis. Tetrahedron Lett. 1995, 36, 8083-8086; (c) Öhberg, L.; Westman, $\mathrm{J}$. An efficient and fast procedure for the Hantzsch dihydropyridine synthesis under microwave conditions. Synlett, 2001, 1296-1298; (d) Agarwal, A.; Chauhan, P. M. S. Solid supported synthesis of structurally diverse dihydropyrido $[2,3-d]$ pyrimidines using microwave irradiation. Tetrahedron Lett., 2005, 46, 1345-1348; (e) Kuraitheerthakumaran, A.; Pazhamalai, S.; Gopalakrishnan M. An efficient and solvent-free one-pot synthesis of 1,4-dihydropyridines under microwave irradiation. Chin. Chem. Lett., 2011, 22,11991202.
Ko, S.; Sastry, M.N.V.; Lin, C.; Yao, C.-F. Molecular iodinecatalyzed one-pot synthesis of 4-substituted-1,4-dihydropyridine derivatives via Hantzsch reaction. Tetrahedron Lett., 2005, 46, 5771-5774.

[11] Sharma, G.V.M.; Reddy, K.L.; Lakshmi, P.S.; Krishna, P.R. In situ Generated $\mathrm{HCl}$ - An efficient catalyst for solvent-free Hantzsch reaction at room temperature: Synthesis of new dihydropyridine glycoconjugates. Synthesis, 2006, 1, 55-58.

[12] Yadav, J.S.; Reddy, B.V.S.; Basak, A.K.; Narsaiah, A.V. Threecomponent coupling reactions in ionic liquids: An improved protocol for the synthesis of 1,4-dihydropyridines.Green Chem., 2003, 5 , 60-63.

[13] Chari, M.A.; Syamasundar, K. Silica gel/ $\mathrm{NaHSO}_{4}$ catalyzed one-pot synthesis of Hantzsch 1,4-dihydropyridines at ambient temperature. Catal. Commun., 2005, 6, 624-626.

[14] Sabitha, G.; Reddy, G.S.K.K.; Reddy, Ch.S.; Yadav, J.S. A novel TMSI-mediated synthesis of Hantzsch 1,4-dihydropyridines at ambient temperature. Tetrahedron Lett., 2003, 44, 4129-4131.

[15] Wang, L.M.; Sheng, J.; Zhang, L.; Han, J.W.; Fan, Z.; Tian, H.; Qian, C.T. Facile $\mathrm{Yb}(\mathrm{OTf})_{3}$ promoted one-pot synthesis of polyhydroquinoline derivatives through Hantzsch reaction. Tetrahedron, 2005, 61, 1539-1543.

[16] (a) Guo, S.; Yuan, Y. One-Pot Synthesis of 1,4-Dihydropyridine and Polyhydroquinoline Derivatives via $L$-Proline catalyzed Hantzsch multicomponent reaction under ultrasound irradiation. Chin. J. Chem., 2010, 28, 811-817; (b) Ruiz, E.; Rodríguez, H.; Coro, J.; Niebla, V.; Rodríguez, A.; Martínez-Alvarez, R.; Novoa de Armas, H.; Suárez, M.; Martín N. Efficient sonochemical synthesis of alkyl 4-aryl-6-chloro-5-formyl-2-methyl-1,4dihydropyridine-3-carboxylate derivatives. Ultrason. Sonochem., 2012, 19, 221-226.

[17] (a) Rovnyak, G.C.; Kimball, S.D.; Beyer, B.; Cucinotta, G.; Dimarco, D.J.; Gougoutas, J.; Hedberg, A.; Malley, M.; McCarthy, J.P.; Zhang, R.; Moreland, S. Calcium entry blockers and activators: Conformational and structural determinants of dihydropyrimidine calcium channel Modulators. J. Med. Chem., 1995, 38, 119-129; (b) Kappe, C.O. Biologically active dihydropyrimidones of the Biginelli-type-a literature survey. Eur. J. Med. Chem., 2000 35, 1043-1052.

[18] Atwal, K.S.; Swanson, B.N.; Unger, S.E.; Floyd, D.M.; Moreland, S.; Hedberg, A.; O'Reilly, B.C. Dihydropyrimidine calcium channel blockers. 3. 3-Carbamoyl-4-aryl-1,2,3,4-tetrahydro-6-methyl-5pyrimidinecarboxylic acid esters as orally effective antihypertensive agents. J. Med. Chem., 1991, 34, 806-811.

[19] (a) Kappe, C.O.; Fabian, W.M.F.; Semones, M.A. Conformational analysis of 4-aryl-dihydropyrimidine calcium channel modulators. A comparison of ab initio, semiempirical and X-ray crystallographic studies. Tetrahedron, 1997, 53, 2803-2816; (b) Kappe, C.O. Recent Advances in the Biginelli dihydropyrimidine synthesis. New tricks from an old dog. Acc. Chem. Res., 2000, 33, 879888; (c) Kappe, C.O.; Shishkin, O.V.; Uray, G.; Verdino, P. X-Ray structure, conformational analysis, enantioseparation, and determination of absolute configuration of the mitotic kinesin Eg5 inhibitor monastrol. Tetrahedron, 2000, 56, 1859-1862; (d) Kappe, C.O. 100 years of the Biginelli dihydropyrimidine synthesis. Tetrahedron, 1993, 49, 6937-6963.

[20] Ranu, B.C.; Hajra, A.; Jana, U. Indium(III)chloride-catalyzed onepot synthesis of dihydropyrimidinones by a three-component coupling of 1,3-dicarbonyl compounds, aldehydes, and urea: An improved procedure for the Biginelli reaction. J. Org. Chem., 2000, 65, 6270-6272.

[21] Atwal, K.S.; Rovnyak, G.C.; Schwartz, J.; Moreland, S.; Hedberg, A.; Gougoutas, J.Z.; Malley, M.F.; Floyd, D.F. Dihydropyrimidine calcium channel blockers: 2-heterosubstituted 4-aryl-1,4-dihydro6-methyl-5-pyrimidine carboxylic acid esters as potent mimics of dihydropyridines. J. Med. Chem., 1990, 33, 1510-1515.

[22] Biginelli, P. Derivati aldeidureidici degli eteri acetil-ed os-salacetico. Gazz. Chim. Ital., 1893, 23, 360-416.

[23] Mandhane, P.G.; Joshi, R.S.; Nagargoje, D.R.; Gill, Ch.H. An efficient synthesis of 3,4-dihydropyrimidin-2(1H)-ones catalyzed by thiamine hydrochloride in water under ultrasound irradiation. Tetrahedron Lett., 2010, 51, 3138-3140.

[24] (a) Manhas, M.S.; Ganguly, S.N.; Mukherjee, S.; Jain, A.K.; Bose, A. K. Microwave initiated reactions: Pechmann coumarin synthesis, Biginelli reaction, and acylation. Tetrahedron Lett., 2006, 47, 2423-2425; (b) Dandia, A.; Saha, M.; Taneja, H. Synthesis of 
fluorinated ethyl 4-aryl-6-methyl-1,2,3,4-tetrahydropyrimidin-2one/thione-5-carboxylates under microwave irradiation. J. Fluor. Chem., 1998, 90, 17-21; (c) Pasunooti, K.K.; Chai, H.; Jensen, Ch.N.; Gorityala, B.K.; Wang, S.; Liu, X-W. A microwave-assisted copper-catalyzed three-component synthesis of dihydropyrimidinones under mild conditions. Tetrahedron Lett., 2011, 52, 80-84; (d) Kidwai, M.; Saxena, S.; Mohan, R.; Venkataramanan R. A novel one pot synthesis of nitrogen containing heterocycles: An alternate methodology to the Biginelli and Hantzsch reactions. $J$. Chem. Soc. Perkin. Trans. 2002, 1, 1845-1846.

[25] Kapoor, K.K.; Ganai, B.A.; Kumar, S.; Andotra, C.S. Antimony(III) chloride impregnated on alumina-An efficient and economical Lewis acid catalyst for one-pot synthesis of dihydropyrimidinones under solvent-free conditions . Can. J. Chem., 2006, 84, 433-437.

[26] (a) Legeay, J.C.; Eynde, J.J.V.; Bazureau, J.P. A new approach to $\mathrm{N}-3$ functionalized 3,4-dihydropyrimidine-2(1H)-ones with 1,2,4oxadiazole group as amide isostere via ionic liquid-phase technology Tetrahedron Lett. 2007, 48, 1063-1068; (b) Desai, B.; Dallinger, D.; Kappe, C.O. Microwave-assisted solution phase synthesis of dihydropyrimidine $\mathrm{C} 5$ amides and esters. Tetrahedron, 2006, 62, 4651-4664.

[27] Maiti, G.; Kundu, P.; Guin, C. One-pot synthesis of dihydropyrimidinones catalysed by lithiumbromide: An improved procedure for the Biginelli reaction. Tetrahedron Lett., 2003, 44, 27572758.

[28] Shaabani, A.; Bazgir, A.; Teimouri, F. Ammonium chloridecatalyzed one-pot synthesis of 3,4-dihydropyrimidin-2-(1H)-ones under solvent-free conditions. Tetrahedron Lett., 2003, 44, 857859.

[29] Salehi, H.; Guo, Q.-X. A facile and efficient one-pot synthesis of dihydropyrimidinones catalyzed by magnesium bromide under solvent-free conditions. Synth. Commun., 2004, 34, 171-179.

[30] Chitra, S.; Pandiarajan, K. Calcium fluoride: An efficient and reusable catalyst for the synthesis of 3,4-dihydropyrimidin-2(1H)-ones and their corresponding $2(1 \mathrm{H})$ thione: An improved high yielding protocol for the Biginelli reaction. Tetrahedron Lett., 2009, 50, 2222-2224.

[31] Lu, J.; Bai, Y.; Catalysis of the Biginelli reaction by ferric and nickel chloride hexahydrates. One-pot synthesis of 3,4dihydropyrimidin-2(1H)-ones. Synthesis, 2002, 466-470.

[32] Kumar, K.A.; Kasthuraiah, M.; Reddy, C.S.; Reddy, C.D. $\mathrm{Mn}(\mathrm{OAc})_{3} \cdot 2 \mathrm{H}_{2} \mathrm{O}$-mediated three-component, one-pot, condensation reaction: An efficient synthesis of 4-aryl-substituted 3,4dihydropyrimidin-2-ones. Tetrahedron Lett., 2001, 42, 7873-7875.

[33] Fu, N.-Y.; Yuan, Y.F.; Cao, Z.; Wang, S.-W.; Wang, J.-T.; Peppe, C. Indium(III)bromide-catalyzed preparation of dihydropyrimidinones: Improved protocol conditions for the Biginelli reaction. Tetrahedron, 2002, 58, 4801-4807.

[34] Jenner, G. Effect of high pressure on Biginelli reactions. Steric hindrance and mechanistic considerations. Tetrahedron Lett., 2004, 45, 6195-6198.

[35] Narsaiah, A.V.; Basak, A.K.; Nagaiah, K. Cadmium chloride: An efficient catalyst for one-pot synthesis of 3,4-dihydropyrimidin2(1H)-ones. Synthesis, 2004, 1253-1256.

[36] Tu, S.; Fang, F.; Miao, Ch.; Jiang, H.; Feng, Y.; Shi, Y.; Wang, X. One-pot synthesis of 3,4-dihydropyrimidin-2(1H)-ones using boric acid as catalyst. Tetrahedron Lett., 2003, 44, 6153-6165.

[37] Debache, A.; Boumoud, B.; Amimour, A.; Belfaitah, A.; Rhouati, S.; Carboni, B. Phenylboronic acid as a mild and efficient catalyst for Biginelli reaction. Tetrahedron Lett., 2006, 47, 5697-5699.

[38] Kalita, H.R.; Phukan, P. CuI as reusable catalyst for the Biginelli reaction. Catal. Commun., 2007, 8, 179-182.

[39] Yadav, J.S.; Reddy, B.V.S.; Srinivas, R.; Venugopal, C.; Ramalingam, T. LiClO-Catalyzed one-pot synthesis of dihydropyrimidinones: An improved protocol for Biginelli reaction. Synthesis, 2001, 1341-1345.

[40] Gohain, M.; Prajapati, D.; Sandhu, J.S. A novel Cu-catalysed threecomponent one-pot synthesis of dihydropyrimidin- $2(1 H)$-ones using microwaves under solvent-free conditions. Synlett, 2004, 235238.

[41] Paraskar, A.S.; DewKar, G.K.; Sudalai, A. $\mathrm{Cu}(\mathrm{OTf})_{2}$ : A reusable catalyst for high-yield synthesis of 3,4-dihydropyrimidin-2(1H)ones. Tetrahedron Lett., 2003, 44, 3305-3308.
[42] Khodaei, M.M.; Salehi, P.; Zolfigol, M.A.; Sirouszadeh, S. Efficient synthesis of 3,4-dihydropyrimidin-2(1H)-ones by aluminum hydrogensulfate. Pol. J. Chem., 2004, 78, 385-388.

[43] Bose, D.S.; Kumar, R.K.; Fatima, L. A remarkable rate acceleration of the one-pot three-component cyclocondensation reaction at room temperature: An expedient synthesis of mitotic kinesin Eg5 inhibitor monastrol. Synlett, 2004, 279-282.

[44] Hu, E.H.; Silder, D.R.; Dolling, U.H. Unprecedented catalytic three component one-pot condensation reaction: An efficient synthesis of 5-alkoxycarbonyl-4-aryl-3,4-dihydropyrimidin-2(1H)-ones. J. Org. Chem., 1998, 63, 3454-3457.

[45] Kappe, C.O. Kumar, D. Varma, R.S.. Microwave assisted highspeed parallel synthesis of 4-aryl-3,4-dihydropyrimidin-2 $(1 H)$-ones using a solventless Biginelli condensation protocol. Synthesis, 1999, 1799-1803.

[46] Tamaddon, F.; Ramzi, Z.; Ali Jafari, A. Synthesis of 3,4dihydropyrimidin-2(1H)-ones and 1,4-dihydropyridines using ammonium carbonate in water. Tetrahedron Lett., 2010, 51, 11871189.

[47] Kolosov, M.A.; Orlov, V.D.; Beloborodov, D.A.; Dotsenko, V.V. A chemical placebo: $\mathrm{NaCl}$ as an effective, cheapest, non-acidic and greener catalyst for Biginelli-type 3,4-dihydropyrimidin-2(1H)ones (-thiones) synthesis. Mol. Divers, 2009, 13, 5-25.

[48] Prodius, D.; Macaev, F.; Mereacre, V.; Shova, S.; Lutsenco, Y.; Styngach, E.; Ruiz, P.; Muraviev, D.; Lipkowski, J.; Simonov, Y.A.; Turta, C. Synthesis and characterization of $\left\{\mathrm{Fe}_{2} \mathrm{CuO}\right\}$ clusters as precursors for nanosized catalytic system for Biginelli reaction. Inorg. Chem. Commun., 2009, 12, 642-645.

[49] (a) Rafiee, E.; Jafari, H. A practical and green approach towards synthesis of dihydropyrimidinones: Using heteropolyacids as efficient catalysts. Bioorg. Med. Chem. Lett., 2006, 16, 2463-2466; (b) Maradur, S.P.; Gokavi, G.S. Heteropolyacid catalyzed synthesis of 3,4-dihydropyrimidin-2(1H)-ones. Catal. Commun., 2007, 8, 279-284.

[50] Salehi, P.; Dabiri, M.; Zolfigol, M.A.; Bodaghi Fard, M.A. Silica sulfuric acid: An efficient and reusable catalyst for the one-pot synthesis of 3,4-dihydropyrimidin-2(1H)-ones. Tetrahedron Lett., 2003, 44, 2889-2891.

[51] Cepanec, I.; Litvić, M.; Bartolinčić, A.; Lovrić, M. Ferric chloride/tetraethyl orthosilicate as an efficient system for synthesis of dihydropyrimidinones by Biginelli reaction. Tetrahedron, 2005, 61, 4275-4280

[52] (a) Debache, A.; Ghalem, W.; Boulcina, R.; Belfaitah, A.; Rhouati, S.; Carboni, B. An efficient one-step synthesis of 1,4dihydropyridines via a triphenylphosphine-catalyzed threecomponent Hantzsch reaction under mild conditions. Tetrahedron Lett., 2009, 50, 5248-5250; (b) Debache, A.; Ghalem, W.; Boulcina, R.; Belfaitah, A.; Rhouati, S.; Carboni, B. Triethylamine promoted efficient synthesis of 3,4-dihydropyrimidin-2(1H)ones/thiones using a solvent-free Biginelli condensation. Lett. Org. Chem., 2010, 7, 272-276. (c) Debache, A.; Amimour, A.; Belfaitah, A.; Rhouati, S.; Carboni, B. A one-pot synthesis of 3,4dihydropyrimidin-2-(1H)-ones/thiones catalysed by triphenylphosphine as Lewis base. Tetrahedron Lett., 2008, 49, 6119-6121.

[53] Shen, Z-L.; Xu, X-P.; Ji, S-J. Bronsted base-catalysed one-pot three-component Biginelli-type reaction: An efficient synthesis of 4,5,6-triaryl-3,4-dihydropyrimidin-2 $(1 H)$-ones and mechanistic study. J. Org. Chem., 2010, 75, 1162-1167.

[54] (a) Eynde, J.J.V.; Delfosse, F.; Mayence, A.; Haverbeke, Y. V. Old reagents, new results: Aromatization of Hantzsch 1,4dihydropyridines with manganese dioxide and 2,3-dichloro-5,6dicyano-1,4-benzoquinone. Tetrahedron, 1995, 51, 6511-6516; (b) Loev, B.; Goodman, M.M.; Snader, K.M.; Tedeschi, R.; Macko, E. Hantzsch-type dihydropyridine hypotensive agents. J. Med. Chem., 1974, 17, 956-965.

[55] (a) Nikpassand, M.; Mamaghani, M.; Tabatabaeian, K. An efficient one-pot three-component synthesis of fused 1,4-dihydropyridines using HY-Zeolite. Molecule, 2009, 14, 1468-1474.

[56] (a) Hong, M.; Cai, Ch.; Yi, W-B. Hafnium (IV) bis(perfluorooctanesulfonyl)imide complex catalyzed synthesis of polyhydroquinoline derivatives via unsymmetrical Hantzsch reaction in fluorous medium. J. Fluor. Chem., 2010, 131, 111-114; (b) Karade, N.N.; Budhewar, V.H.; Shinde, S.V.; Jadhav, W.N. LProline as an efficient organo-catalyst for the synthesis of polyhydroquinoline via multicomponent Hantzsch reaction. Lett. Org. 
Chem., 2007, 4, 16-19; (c) Donelson, J.L.; Gibbs, R.A.; De, S.K. An efficient one-pot synthesis of polyhydroquinoline derivatives through the Hantzsch four component condensation. J. Mol. Catal. A: Chem., 2006, 256, 309-311.

[57] (a) Debache, A.; Boulcina, R.; Tafer, R.; Belfaitah, A.; Rhouati, S.; Carboni, B. Ca(NO3) $)_{2} 4 \mathrm{H}_{2} \mathrm{O}$-catalysed Biginelli Reaction: One-pot Synthesis of 1,2,3,4-Tetrahydropyrimidin-2-ones/pyrimidine-2thiones under Solvent-free Conditions. Chin. J. Chem., 2008, 26, 2112-2116; (b) Ramalingan, C.; Kwak Y.-W. Tetrachlorosilane catalyzed multicomponent one-step fusion of biopertinent pyrimidine heterocycles Tetrahedron, 2008, 64, 5023-5031.

[58] a) Shaabani, A.; Sarvary, A.; Rahmati A.; Rezayan, A.H. Ionic Liquid/Silica Sulfuric acid promoted fast synthesis of a BiginelliLike Scaffold Reaction. Lett. Org. Chem., 2007, 4, 68-71; b) Lin, H.; Zhao, Q.; Xu, B.; Wang, X. Nafion-H catalyzed cyclocondensation reaction for the synthesis of octahydroquinazolinone derivatives. J. Mol. Catal. A: Chem., 2007, 268, 221-226.

(C) Debache et al.; Licensee Bentham Open.

This is an open access article licensed under the terms of the Creative Commons Attribution Non-Commercial License (http://creativecommons.org/licenses/ by-nc/3.0/) which permits unrestricted, non-commercial use, distribution and reproduction in any medium, provided the work is properly cited. 